\title{
Localization of millimeter size defects in a steel hemispherical shell
}

\section{Veira Canle, Daniel}

IEEE

2019

Veira Canle , D , Mäkinen , J , Gritsevich , M , Salmi , A \& Haeggström , E 2019 , Localization of millimeter size defects in a steel hemispherical shell . in 2019 IEEE International Ultrasonics Symposium (IUS) . IEEE International Ultrasonics Symposium, IEEE , pp. 1575-1578, IEEE International Ultrasonics Symposium , Glasgow , United Kingdom , 06/10/2019 . https://doi.org/10.1109/ULTSYM.2019.8925746

http://hdl.handle.net/10138/337242

https://doi.org/10.1109/ULTSYM.2019.8925746

unspecified

acceptedVersion

Downloaded from Helda, University of Helsinki institutional repository.

This is an electronic reprint of the original article.

This reprint may differ from the original in pagination and typographic detail.

Please cite the original version. 
(C) 2019 IEEE. Personal use of this material is permitted. Permission from IEEE must be obtained for all other uses, in any current or future media, including reprinting/republishing this material for advertising or promotional purposes, creating new collective works, for resale or redistribution to servers or lists, or reuse of any copyrighted component of this work in other works. 


\title{
Localization of millimeter size defects in a steel hemispherical shell
}

\author{
Daniel Veira Canle ${ }^{1}$, Joni Mäkinen ${ }^{1}$, Maria Gritsevich ${ }^{1,2}$, Ari Salmi ${ }^{1}$, Edward Hæggström ${ }^{1}$ \\ ${ }^{1}$ Electronics Research Laboratory, Physics Department, University of Helsinki, Finland \\ ${ }^{2}$ Institute of Physics and Technology, Ural Federal University, Mira str. 19, 620002 Ekaterinburg, \\ Russia
}

\begin{abstract}
Ultrasonic NDT is a commonly used modality for ensuring structural integrity. When the geometry of the structure in question is complex, challenges arise, especially for structures that curve upon themselves, such as pipes and spheres. One challenging case is the hemisphere, where edge reflections (such as occurring in plates) and multiple propagation paths (such as in spheres) add complexity to the task. We propose a contactless method based on laser ultrasound to generate guided waves on a $50 \mathrm{~mm}$ in diameter stainless-steel hemispherical shell. We studied samples featuring holes of various diameters and determined the detectability limit of our technique.
\end{abstract}

\section{INTRODUCTION}

Guided waves are important in structural health monitoring since they can propagate large distances with little attenuation [1][2][3]. Typically, these waves are generated and detected by contact transducers. These elements often require coupling to minimize the loss of acoustic energy. Their large footprint integrates the propagating wave across the detection area leading to a loss of resolution. Furthermore, contacting transducers load the sample which causes wave distortion. These drawbacks can be circumvented by laser ultrasound [4]. This contactless technique allows for rapid sample inspection with high spatial resolution.

Structures featuring rotational symmetry pose challenges when examined with acoustic waves. Not only do the phase velocity, amplitude profile, and cut-off frequency change for different guided wave modes but there are also multiple propagation paths from the detection to the pickup point [5]. This causes wave interference and loss of contrast [6].

Typically, when exciting acoustic waves with a laser, an elastodynamic point source is assumed [7]. The propagating waves travel radially outwards from the excitation point. This propagation pattern poses a challenge in structures with rotational symmetry. The waves embrace the structure leading to circumferential waves and interference. This can be alleviated by applying directionality, which can be achieved by means of a laser line source for acoustic wave generation. These directed acoustic waves reflect off defects present in the structure allowing one to determine their size and position.
We determined the detectability limit of the directional technique. This was achieved by studying samples featuring holes of different size and by comparing the experimental results to those obtained with finite element method (FEM) simulations.

\section{METHODS}

To understand guided wave propagation on the shell, we performed FEM simulations on a model of the steel hemisphere. These simulations were verified experimentally by laser ultrasound measurements. In our experiments, the samples rotated azimuthally and a Nd:YAG laser line source (CFR Big Sky Laser Series, 8 ns, 40 mJ 3 Hz PRF) generated a directed acoustic field at the apex of the sample (Fig.1).

A cylindrical lens $80 \mathrm{~mm}$ in focal length projected the Nd:YAG laser into a line. Guided acoustic waves propagated in a direction that is perpendicular to the line excitation. The lens was fixed to the table while the sample rotated azimuthally in steps of 1.6 degrees allowing a full scan of the sample.

A laser Doppler vibrometer (Polytec OFV303) in displacement mode positioned $0.8 \mathrm{~mm}$ from the edge of the sample detected the propagating waves and a Picoscope 3000 series (50 MHz bandwidth) stored the data into a computer.

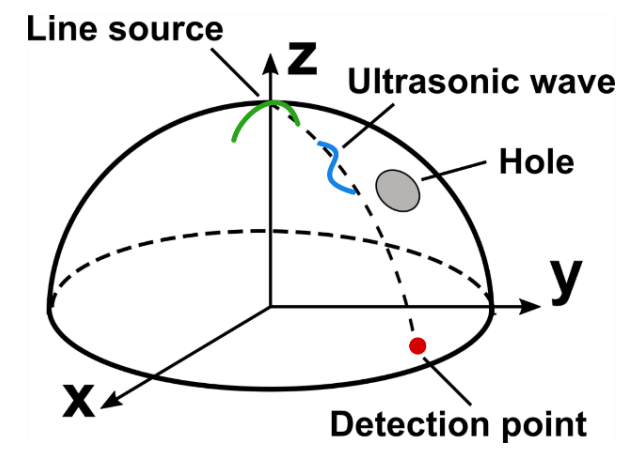

Fig 1. Sample diagram. A line source (green line) excited an ultrasonic wave (blue line) that travelled along a preferred direction (dashed line). An LDV detected the propagating waves (red dot).

We measured four different samples. They consisted of stainless-steel hemispherical shells $50 \mathrm{~mm}$ in diameter and $0.6 \mathrm{~mm}$ thick. These structures were polished to a mirror like finish to reflect as much light back as possible to the interferometer and to ensure a large SNR. The shells were glued onto a hemispherical holder so that one drop of Loctite 
® 3090 held them in place at the apex. The holders were such that a $3 \mathrm{~mm}$ wide section from the brim of the steel shell was left exposed. We placed the detection spot on this ribbon of exposed steel at $0.8 \mathrm{~mm}$ from the brim of the sample.

One sample was pristine and the others featured a hole at a polar angle $\theta=45^{\circ}$ and azimuthal angle $\varphi=90^{\circ}$. The holes varied in size; they were $\varnothing=9.5 \mathrm{~mm}, 4 \mathrm{~mm}$, and $2 \mathrm{~mm}$.

FEM simulations were done with COMSOL Multiphysics $^{\circledR}$ [8]. Previously we have modelled laser excitation induced Lamb wave propagation in a hemispherical steel shell [9]. In this work we continued these simulations and compared the results to experimental data.

The simulations use COMSOL's Solid Mechanics and Heat Transfer modules to compute the elastic wave propagation and the heating from a laser source in time domain. To model the generation of elastic waves by laser heating, these two modules are combined with the predefined multiphysics coupling for thermal expansion. A small difference in the mechanics of laser excitation arises in the simulations, compared to the experiments, as forces from ablation are neglected. The line source in the simulations is defined as two perpendicular gaussian boundary heat sources with FWHM= $0.90 \mathrm{~mm}$ and $7.80 \mathrm{~mm}$. Meshing and time stepping were done such that a $2 \mathrm{MHz}$ longitudinal wave was resolved. This was found to be sufficient to solve the Lamb waves in the frequency range of interest. The mesh size was $\lambda / 6$ and the time step was $1 /(60 f)$, where $\lambda=c / f, c=5780 \mathrm{~m} / \mathrm{s}$ and $f=2 \mathrm{MHz}$. The mesh was refined in the region where heating was applied. Multiple simulations were done for each sample where each simulation set covered a $90^{\circ}$ rotation of the sample in $1.6^{\circ}$ steps. From one $90^{\circ}$ data set a full $360^{\circ}$ data set corresponding to the measurements was obtained by exploring symmetries. The symmetries cut the simulation time by a factor of four.

To determine the defect diameter, we located the regions where the signal squared falls by $-3 \mathrm{~dB}$. The boundaries of these regions are marked with white dashed lines in Figs. $2 \& 3$. The obtained angle can then be used to estimate the defect size (Eq. 1).

$$
\mathrm{D}=\Delta \phi \mathrm{R}[1]
$$

where $\mathrm{D}$ is the defect diameter, $\Delta \Phi$ the azimuthal angle difference in radians, and $\mathrm{R}$ the radius of the shell.

When the quasi-antisymmetric mode is reflected off the defect, echoes radiate from it (Figs. $2 \& 3$ echoes 1,2 ). The distance from the edge of the sample to the defect is (Eq. 2):

$\mathrm{d}=\Delta \mathrm{tv}_{\mathrm{A} 0}+\mathrm{d}_{\mathrm{LDV}}[2]$

Here $\Delta t$ is the time difference between the arrival of the echo radiating from the defect and the ballistic quasiantisymmetric Lamb wave.

To calculate the phase velocity of the quasi-antisymmetric mode we determined the time-of-flight of said wave front from the experimental data of the intact case (Eq. 3).
$\mathrm{v}_{\mathrm{A}_{0}}=\frac{\left(\frac{\pi}{2} R-\mathrm{d}_{\mathrm{LDV}}\right)}{\mathrm{t}_{\mathrm{A}_{n}}}$ [3]

$\mathrm{v}_{\mathrm{A0}}=2946 \pm 137 \mathrm{~m} \mathrm{~s}^{-1}$

In Eq. $3 \mathrm{t}_{\mathrm{A}_{\mathrm{n}}}$ is the time-of-flight of the fastest component of the quasi-antisymmetric Lamb wave.

\section{RESULTS}

The results from the experiments and simulations are presented in Figs. 2\&3. The dashed lines highlight the width of the wave front that is bent by the flaw in the structure. Echoes at $90^{\circ}$ and $270^{\circ}$ arise from the near and the far edge of the hole and are used to determine the distance to the defect.

Table 1 collects the results from the experiments and the FEM simulations.
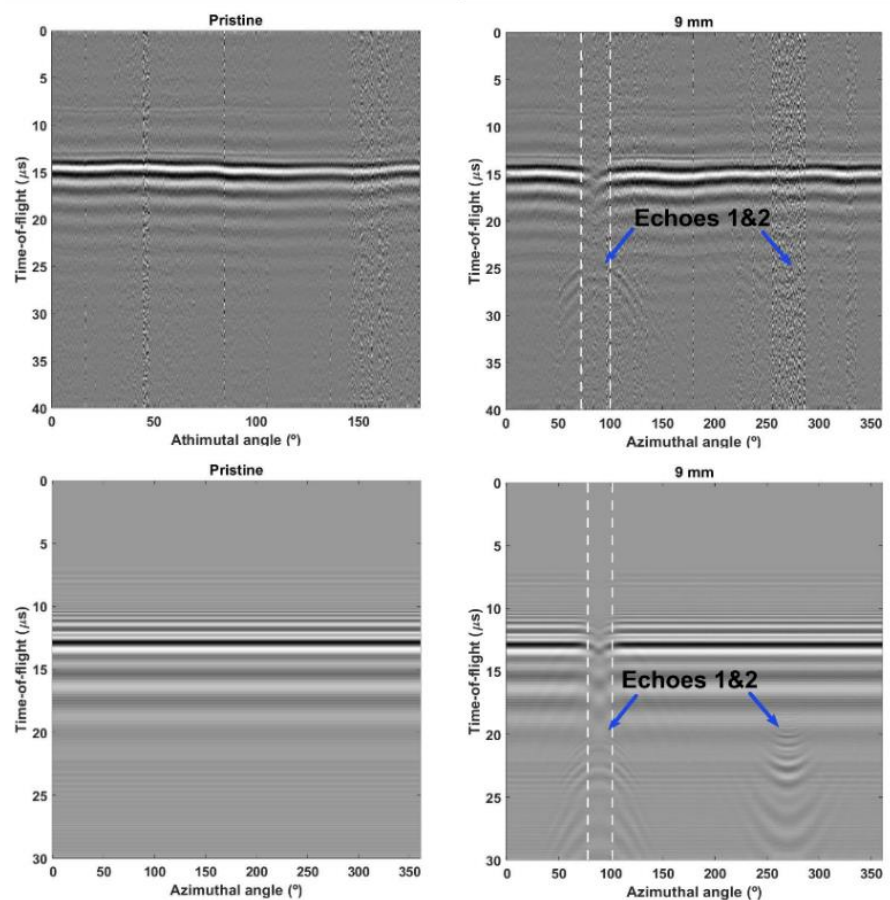

Figure 2. Comparison of pristine sample (left) and a structure featuring a $9.5 \mathrm{~mm}$ hole (right).

\begin{tabular}{|l|l|l|}
\hline \multicolumn{3}{|l|}{ Experimental Results } \\
\hline Sample & Diameter $(\mathrm{mm})$ & Distance $(\mathrm{mm})$ \\
\hline $9.5 \mathrm{~mm}$ & $9.4 \pm 2.8$ & $17.56 \pm 1.4$ \\
\hline $4 \mathrm{~mm}$ & $4.9 \pm 2.8$ & $19.63 \pm 1.4$ \\
\hline $2 \mathrm{~mm}$ & - & $20.0 \pm 1.4$ \\
\hline Simulation Results & \multicolumn{2}{|l|}{} \\
\hline Sample & Diameter $(\mathrm{mm})$ & Distance $(\mathrm{mm})$ \\
\hline $9.5 \mathrm{~mm}$ & $9.47 \pm 2.2$ & $15.56 \pm 1.4$ \\
\hline $4 \mathrm{~mm}$ & $3.94 \pm 2.2$ & $17.82 \pm 1.4$ \\
\hline $2 \mathrm{~mm}$ & - & $18.33 \pm 1.4$ \\
\hline
\end{tabular}

Table 1. Experimental and simulation results. The magnitudes feature confidence limits of one standard deviation. 

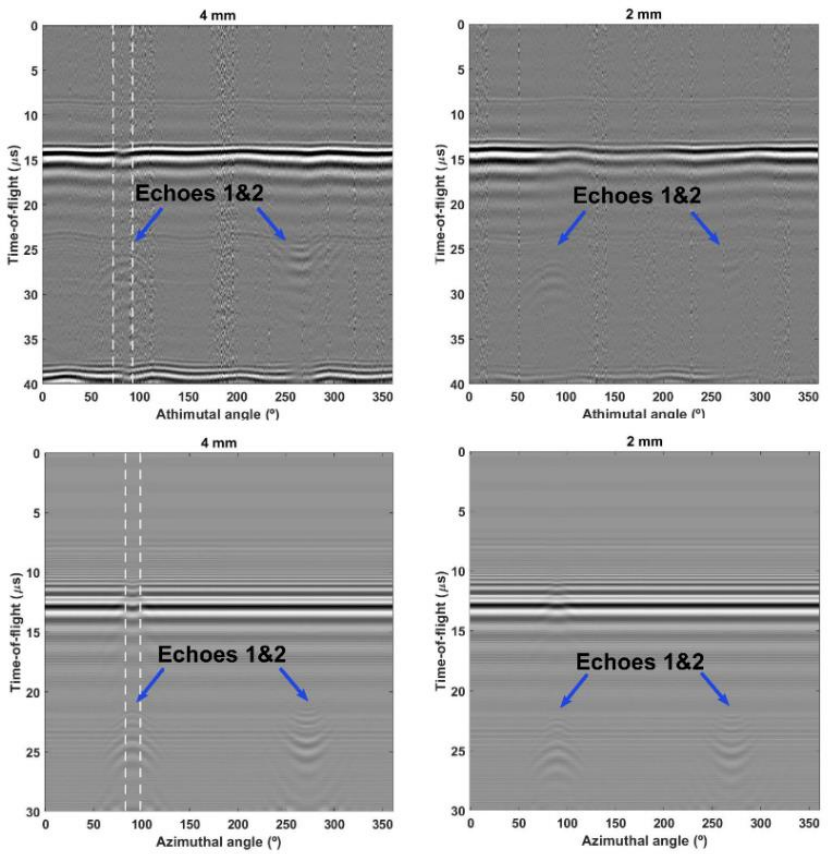

Figure 3. Comparison of a sample featuring a $4 \mathrm{~mm}$ defect (left) and a sample with a $2 \mathrm{~mm}$ hole (right). In the $2 \mathrm{~mm}$ case the defect behaves like an acoustic point source and it is not possible to determine its size, only its location. The grainy regions in the figure correspond to locations where there was poor SNR.

\section{DISCUSSION}

Figures $2 \& 3$ show that the curvature of the echoes arising from the defect changes from convex at $\varphi=90^{\circ}$ to concave at $\varphi=270^{\circ}$. In the former case the propagating waves launched by the line excitation are bent by the defect. In the latter case these reflect off the hole yielding patters with a concave shape.

The defect bends the propagating quasi-antisymmetric mode which can be used to determine the size of the scatterer. The effect is less pronounced the smaller the defect is, until the scatterer becomes a point source of elastic waves. This is seen in Fig. 3 for the $2 \mathrm{~mm}$ hole.

The central frequency of the propagating wave package is $800 \mathrm{kHz}$ and the frequency bandwidth of the interferometer is $2 \mathrm{MHz}$. Thus, considering our phase velocity $3056 \mathrm{~m} \mathrm{~s}^{-1}$ yields a central wavelength of $\lambda=3.8 \mathrm{~mm}$ with an upper limit $\lambda=1.6 \mathrm{~mm}$. In Fig 3, it is apparent that the defect detection limit is between 2 and $4 \mathrm{~mm}$, thus in the same order of magnitude as the wavelength of the wave package.

The curvature modifies the cut-off frequencies of the propagating guided waves according [5]. For a curved aluminium plate, these cut-off frequencies start differing significantly from the plate model for a thickness to radius ratio of 0.024 .

$\frac{\mathrm{d}}{\mathrm{R}}>0.024[4]$ where $d$ is the sample thickness and $r$ the radius of curvature of the structure. In our case, the wavelength of the wave package is between $\lambda=1.6 \mathrm{~mm} \sim 2 d$ and $\lambda=3.8 \mathrm{~mm} \sim 3 d$. Solving Eq. 4 for R yields.

\section{$11.1 \mathrm{~mm}>R>26.4 \mathrm{~mm}$}

The fact that in our case $\mathrm{R}=25 \mathrm{~mm}$ means that the propagation of long wavelength components is affected by the curvature of the structure while the short wavelength contribution is not.

The detection limit is set by the diffraction of the acoustic field. The fact that we reached this limit with a directed acoustic field means that one can perform non-destructive evaluation on curved structures in a similar way as in plates.

\section{CONCLUSION}

We introduced a laser ultrasound method to detect and size flaws on hemispherical shells.

\section{REFERENCES}

[1] C. Valle, M. Niethammer, J. Qu, and L. J. Jacobs, "Crack characterization using guided circumferential waves," J. Acoust. Soc. Am., vol. 110, no. 3, pp. 1282-1290, Sep. 2001.

[2] Z. Li, C. He, Z. Liu, and B. Wu, "Quantitative detection of lamination defect in thin-walled metallic pipe by using circumferential Lamb waves based on wavenumber analysis method," NDT E Int., 2019.

S. Towfighi and T. Kundu, "Elastic wave propagation in anisotropic spherical curved plates," Int. J. Solids Struct., vol. 40, no. 20, pp. 5495-5510, Oct. 2003.

[4] C.B Scruby and L.E Drain, Laser ultrasonics, techniques and applications, vol. 24, no. 3. 2003.

[5] Ka Lok Jimmy Fong, "A Study of Curvature Effects on Guided Elastic Waves," Imperial College London, 2005.

[6] H. Kim, K. Jhang, M. Shin, and J. Kim, “A noncontact NDE method using a laser generated focused-Lamb wave with enhanced defect-detection ability and spatial resolution," NDT E Int., vol. 39, no. 4, pp. 312-319, Jun. 2006.

[7] M. Kley, C. Valle, L. J. Jacobs, J. Qu, and J. Jarzynski, "Development of dispersion curves for two-layered cylinders using laser ultrasonics," $J$. Acoust. Soc. Am., vol. 106, no. 2, pp. 582-588, Aug. 1999. 
[8] S. COMSOL AB, Stockholm, "COMSOL Multiphysics ${ }^{\circledR}$ v. 5.4.” 2019.

[9] J. Mäkinen, D. Veira Canle, R. Blomqvist, A. Salmi, and E. Haeggström, "Simulation of Laser-excited

Surface Acoustic Waves Traveling on A

Hemispherical Steel Shell," 2018. 